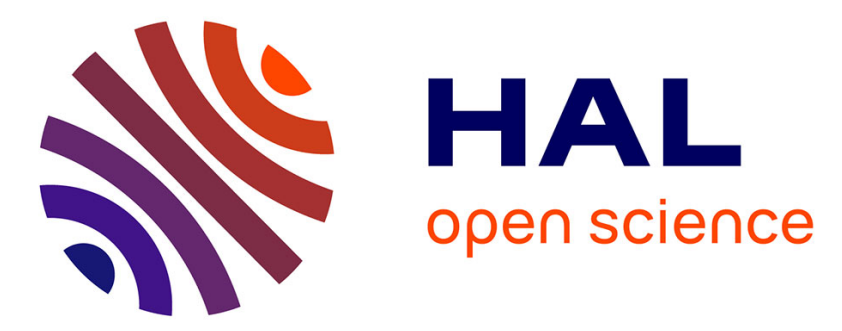

\title{
Optimization study of a piezoelectric bistable generator with doubled voltage frequency using harmonic balance method
}

\author{
Weiqun Liu, Fabien Formosa, Adrien Badel
}

\section{- To cite this version:}

Weiqun Liu, Fabien Formosa, Adrien Badel. Optimization study of a piezoelectric bistable generator with doubled voltage frequency using harmonic balance method. Journal of Intelligent Material Systems and Structures, 2017, 28 (5), pp.671 - 686. 10.1177/1045389x16657203 . hal-01901535

\section{HAL Id: hal-01901535 \\ https://hal.science/hal-01901535}

Submitted on 23 Oct 2018

HAL is a multi-disciplinary open access archive for the deposit and dissemination of scientific research documents, whether they are published or not. The documents may come from teaching and research institutions in France or abroad, or from public or private research centers.
L'archive ouverte pluridisciplinaire HAL, est destinée au dépôt et à la diffusion de documents scientifiques de niveau recherche, publiés ou non, émanant des établissements d'enseignement et de recherche français ou étrangers, des laboratoires publics ou privés. 


\title{
Optimization study of a piezoelectric bistable generator with doubled voltage frequency using harmonic balance method
}

\author{
Weiqun Liu ${ }^{1}$, Fabien Formosa ${ }^{2 *}$, Adrien Badel ${ }^{2}$ \\ ${ }^{1}$ School of Mechanical Engineering, Southwest Jiaotong University, 610031, Chengdu, China \\ ${ }^{2}$ Laboratoire SYMME, Université Savoie Mont Blanc, 74000, Annecy, France \\ * Corresponding author : fabien.formosa@univ-smb.fr
}

\begin{abstract}
Bistable generator for vibration energy harvesting is one of the most promising solutions to reach practical enhanced performance. Due to the BSM (Buckled-Spring-Mass) specific behavior, the deformation of the piezoelectric transducer is nonlinearly dependent on the displacement, especially in the inter-well motion case. An analytical model is developed using harmonic balance analysis for the BSM generator architecture. Contrary to the usual method, a special doubled frequency voltage solution in the inter-well motion case is assumed in harmonic balance analysis to obtain an accurate predictive model, which is validated by experimental results. The influence of five critical parameters on the performance is thoroughly discussed. Design rules are then deduced: low damping ratio, properly high coupling level, matched load, optimal buckling level and low characteristic frequency are required to get optimal performance in the inter-well motion case. Besides, we show some interesting results about the parameter optimization study.
\end{abstract}

Keywords: Energy harvesting; Bistable oscillator; Analytical model; Harmonic balance; Optimization

\section{Introduction}

The building of smart city promotes the rapid development and wide applications of WSNs (Wireless Sensor Networks). Good prospects have been foreseen in many facets of the future society, including industry, health, agriculture, scientific research (Ruiz-Garcia et al., 2009; Oliveira et al., 2011; Ko et al., 2010) etc. The energy autonomous requirements for WSNs are being more and more important since people expect as less interference in daily activities as possible. Maintenance cost reduction and lifespan extension are highly concerned. The advancements in energy harvesting technology have confirmed it as the most promising solution to the energy autonomy of WSNs. Among the numerous available wasted energy sources, vibration energy exhibits some good features of relatively high power density and pervasive existence.

Continuous progress has been made in vibration energy harvesting during recent decades (Toprak et al., 2014; Harb, 2011). At the early stage, most of vibration generators were linear with narrow operation band (Roundy et al., 2003; Xu et al., 2012; Arroyo et al., 2013). However, the majority of environment real vibrations are variable and broadband (Neri et al., 2012). Then, wideband vibration harvesting solutions are going through increasing developments as they are capable of providing stable and robust performance. Researchers have proposed a variety of mechanisms (Tang et al., 2010; Zhu et al., 2010): resonance tuning solutions (Leland and Wright, 2006; Roundy and Zhang, 2005; Challa et al., 2011), multi-modal generators (Shahruz, 2006; Yang and Yang, 2009), frequency-up-conversion methods (Pillatsch et al., 2012; Galchev et al., 2011; Wickenheiser and Garcia, 2010), nonlinear generators (Stanton et al., 2009; Sebald et al., 2011; Nguyen and Halvorsen, 2011) and other concepts. 
As one of the numerous effective and promising mechanisms, nonlinear generators have attracted great interests recently. Various architectures were developed, such as piecewise generators (Soliman et al., 2008; Liu et al., 2012), monostable softening or hardening generators (Mann and Sims, 2009; Hajati and Kim, 2011), bistable generators (Liu et al., 2013a; Liu et al. 2014; Masana and Daqaq, 2011; Cottone et al., 2012; Liu et al., 2015; Tang et al., 2011; Cottone et al., 2009; Mann and Owens, 2010; Stanton et al., 2010; Erturk and Inman, 2011), tri-stable generators (Kim and Seok, 2014) etc. Among them, the bistable generator is considered as a most promising one because of its unique features. The snap-through mechanism in the case of large excitations induces large motion amplitude. The frequency-up-conversion effect for the low frequency excitation and the nonlinear wideband response can significantly enhance the harvested power and the operation band.

Although many bistable generators have been published (Harne and Wang, 2013a; Pellegrini et al., 2013), they can be classified into two categories according to the bi-stability mechanisms: i) mechanical buckling effect (Liu et al., 2013a; Liu et al. 2014; Masana and Daqaq, 2011; Cottone et al., 2012; Liu et al., 2015); ii) magnetic interaction (Tang et al., 2011; Cottone et al., 2009; Mann and Owens, 2010;Stanton et al., 2010; Erturk and Inman, 2011).

Until now, numerical simulation and experimental test are two common ways for the investigation of the bistable generator seen in the literature. They usually require significant computation or test time while the results are highly sensitive to the initial states. An overall understanding of the bistable generator's properties and available solutions is usually missing. Consequently, the optimization of the bistable generator becomes somehow difficult and cumbersome. Analytical approach is a good way of addressing this issue. However, only few efforts about modeling and optimization with analytical methods have been performed in the field of nonlinear energy harvesting. Stanton et al. investigated the dynamics of a bistable cantilever generator realized with magnetic interactions using harmonic balance analysis for a generic parameter study (Stanton et al., 2012a) and Melnikov method for a simplified predictive criterion about the homoclinic bifurcation (Stanton et al., 2012b) meanwhile. Owen and Mann investigated the linear and nonlinear electromagnetic coupling models of the harvester with the harmonic balance approach (Owen and Mann, 2012). Harne et al. also utilized harmonic balance method for analyzing a bistable harvester with an auxiliary linear oscillator (Harne et al., 2013b) and subsequently studying the fundamental and super-harmonic effects in bistable energy harvesting (Harne and Wang, 2014). Neiss et al. proposed analytical expressions for the jump-up and jump-down points of monostable nonlinear generators (Neiss et al., 2014) through harmonic balance method. More recently, Leadenham and Erturk took advantage of the harmonic balance approach for developing an analytical model for a nonlinear M-shape harvester (Leadenham and Erturk, 2015) while Chen and Jiang employed the multi-scale method for studying the internal resonance of a bistable harvester with an additional linear oscillator (Chen and Jiang, 2015). However, except comparisons with numerical simulations, experiments did not completely confirm the analytical model accuracy in usual cases. These studies do not analyze the performance dependency on the bistable generator's parameters thoroughly and discuss the optimization issue in depth as well. Moreover, these analytical models are usually derived on the assumption of linear coupling force. Consequently, the present analytical models in the literature are not applicable for the BSM generator because of the nonlinear coupling force due to its special architecture.

In order to propose an improved analytical model for the BSM (Buckled-Spring-Mass) generator architecture recently introduced by the authors (Liu et al., 2013a), a proper perturbation method is to 
be determined. Among different approaches, the straightforward expansion and the Linstedt-Poincaré method are incapable of excluding secular terms while the method of averaging is likely to have inconsistent results in some cases (Nayfeh AH and Mook DT, 2008). Meanwhile, considering that the multi-scale approach usually requires complex operations, the harmonic balance method is considered as a better choice due to the simplicity and the consistency with the real bistable harvester, since some prior knowledge about the solutions can be known in advance from the literature or primary experiments. The frequent utilization (Stanton et al., 2012a; Owen and Mann, 2012; Harne et al., 2013b; Harne and Wang, 2014; Neiss et al., 2014; Leadenham and Erturk, 2015; Chen and Jiang, 2015) justifies the use of the harmonic balance method as well. Therefore, the harmonic balance method is selected for the modeling of the bistable generator. A new specific model is then established in this paper. The model is firstly validated using experimental results. Following that, the influence of the critical parameters on the performance is studied. Then, the average power for the full frequency range in the inter-well motion case is used as a figure of merit to aim at the best available performance of the generator through the optimization study with the proposed analytical model.

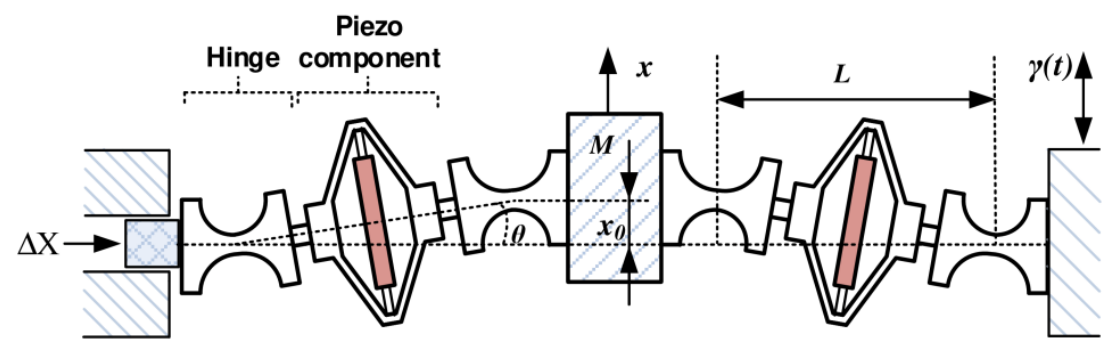

Figure 1. Scheme of the BSM generator.

\section{Bistable generator model}

As presented in figure1, the BSM system is composed of two piezoelectric components acting as electromechanical springs and a central dominant inertial mass respectively. Four flexible hinges are used as rotation parts in the structure and each one can be viewed as the combination of an ideal revolute joint and a rotational spring with the stiffness $K_{\theta}$. The rotation center is placed at the middle point of the hinge. Different from many reported bistable oscillators, the piezoelectric transducers operate in $\mathrm{d}_{33}$ mode, ensuring a high electromechanical coupling level. Table 1 lists the BSM parameters.

Tab. 1. Parameters' definitions for the BSM generator

\begin{tabular}{ll}
\hline Definition & Symbol \\
\hline Mass & $M$ \\
Horizontal Distance between two adjacent rotary centers & $L$ \\
Stiffness of one piezoelectric spring & $K / 2$ \\
Damping coefficient & $\mu$ \\
Capacitance of two parallel piezoelectric elements & $C_{0}$ \\
Piezoelectric force factor & $\alpha$ \\
Rotational stiffness of hinge & $K_{\theta}$ \\
Initial buckled position & $x_{0}$ \\
Electrical load resistance & $R$ \\
\hline
\end{tabular}


Before utilization for harvesting, all the components are assembled and aligned horizontally with the centerline $x=0$ without deformation. We denote this initial free length between two adjacent rotary centers of the piezoelectric element by $l_{0}$. With a small tuning displacement applied at the left, the piezoelectric transducers are compressed while the mass does not move at first due to the rotational stiffness of the flexible hinges before the happening of buckling. As the tuning displacement increases to $\Delta X$, the hinges buckle and the mass moves up or down to one of the two symmetric stable positions $\left(x_{0}\right.$ or $\left.-x_{0}\right)$. Then we have: $l_{0}=L+\Delta X$. Easy to find, the pre-strain of the piezoelectric elements induced by the rotational stiffness is

$$
\Delta s=\sqrt{L^{2}+x_{0}^{2}}-l_{0}
$$

while the variation of the length between the two adjacent rotary centers during operation is calculated by:

$$
\Delta L=\sqrt{L^{2}+x^{2}}-\sqrt{L^{2}+x_{0}^{2}}
$$

Therefore, the deformation of the piezoelectric transducer is written as the function of the mass position $x$ :

$$
\Delta l=\Delta s+\Delta L=-l_{0}+\sqrt{L^{2}+x^{2}}
$$

With a short version of the model of the BSM generator given hereafter, more details can be found in a previous article (Liu et al., 2013a). The classical Euler-Lagrange approach is applied to deduce the dynamic equation. Neglecting any other inertias apart from the central inertial mass considered, the kinetic energy of the system is:

$$
T=\frac{1}{2} M \dot{x}^{2}
$$

We can also write the expression of the total potential energy as follows:

$$
U=\frac{K}{2} \Delta l^{2}+4 \times \frac{1}{2} K_{\theta} \theta^{2}
$$

in which $\theta \approx x / L$. The first term in eq. (5) represents the potential energy of the transducers while the second term comes from the flexible hinges.

The Euler-Lagrange equation is then written as:

$$
\frac{d}{d t}\left(\frac{\partial(T-U)}{\partial \dot{x}}\right)-\frac{\partial(T-U)}{\partial x}=M \ddot{x}+K\left(1-\frac{l_{0}}{\sqrt{L^{2}+x^{2}}}\right) x+4 K_{\theta} \frac{x}{L^{2}}=M \gamma(t)-\alpha V_{p} \sin \theta-\mu \dot{x}
$$

where $V_{p}$ is the piezoelectric voltage.

The electric charge balance equation is:

$$
I=-\alpha \Delta \dot{l}-C_{0} \dot{V}_{p}=\alpha \frac{x \dot{x}}{\sqrt{L^{2}+x^{2}}}-C_{0} \dot{V}_{p}
$$

in which $I$ is the piezoelectric transducer's output current going to the interface circuit. Combining eq. (6) and eq. (7), the full electromechanical equation set is: 


$$
\left\{\begin{array}{l}
M \gamma(t)=M \ddot{x}-\frac{K x_{0}^{2}}{2 L^{2}} x+\frac{K x^{3}}{2 L^{2}}+\mu \dot{x}+\alpha V_{p} \frac{x}{L} \quad\left(x_{0}=\sqrt{\left.l_{0}^{2}-L^{2}-\frac{8 K_{\theta}}{K}\right)}\right. \\
I=\frac{\alpha x \dot{x}}{L}-C_{0} \dot{V}_{p}
\end{array}\right.
$$

The simplification is carried out here using the Taylor expansion (assuming $x<<L$ ). The BSM generator has two stable equilibrium positions $x=x_{0}$ and $x=-x_{0}$ and one unstable equilibrium position $x=0$ providing that $K_{\theta}<\left(l_{0}^{2}-L^{2}\right) K / 8$ is satisfied. It means that the hinge's stiffness determines the threshold of the buckling. Then the simplified model shown in figure 2 (a) can represent the BSM structure after buckled.

From figure 2 (a), it is interesting to notice that the transducers' deformation $\Delta l$ is not proportional to the displacement $x$, which is typical in many regular generators. Instead, a relatively complex nonlinear relationship between $\Delta l$ and $x$ exists as shown in eq. (3). Accordingly, the electrical current generated by the transducers is not only related to the velocity $\dot{x}$ but also to the position $x$ as indicated by the electric equation in eq. (7). Moreover, because of the special symmetric structure of the BSM generator, the deformation $\Delta l$ is identical for position $x$ and $-x$. For better understanding, the potential and the deformation curves are plotted in figure 2 (b).

Using the parameters defined in Tab.2, eq. (8) is normalized as:

$$
\left\{\begin{array}{l}
\bar{\gamma}(t)=\ddot{\bar{x}}-\frac{1}{2} \omega_{0}^{2} \bar{x}+\frac{1}{2} \frac{\omega_{0}^{2}}{\varepsilon^{2}} \bar{x}^{3}+2 \xi \omega_{0} \dot{\bar{x}}+\frac{k^{2}}{1-k^{2}} \frac{\omega_{0}^{2} \bar{x}}{\varepsilon} \bar{V} \\
\bar{I}=\frac{\bar{x} \dot{\bar{x}}}{\varepsilon}-\dot{\bar{V}}
\end{array}\right.
$$

Using the normalized parameters, the generator's power output is:

$$
P=V_{p} I=\frac{\alpha^{2} x_{0}^{2}}{C_{0}} \overline{V I}=M L^{2} \frac{k^{2} \omega_{0}^{2}}{1-k^{2}} \overline{V I}=M L^{2} \bar{P}
$$

Then the product of the output power $P$ and the efficiency of the interface circuit determine the final harvested power. For the case of a pure resistor load, the circuit efficiency would be $100 \%$ and $P$ is therefore the harvested power.

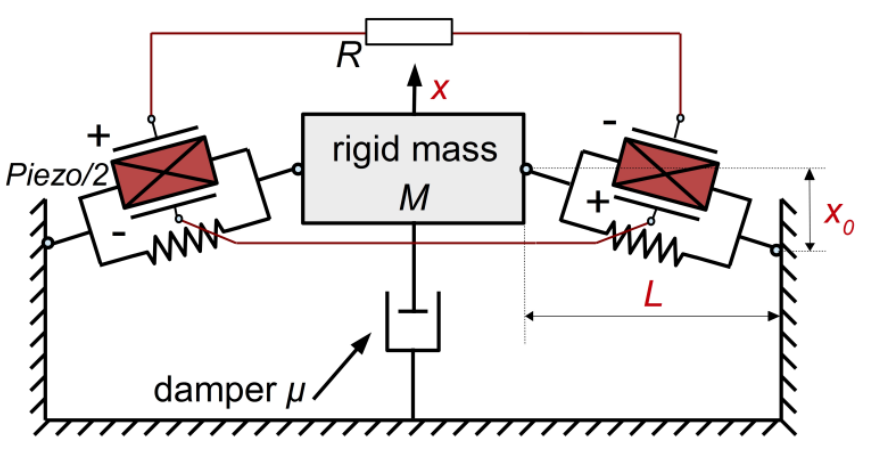

(a)

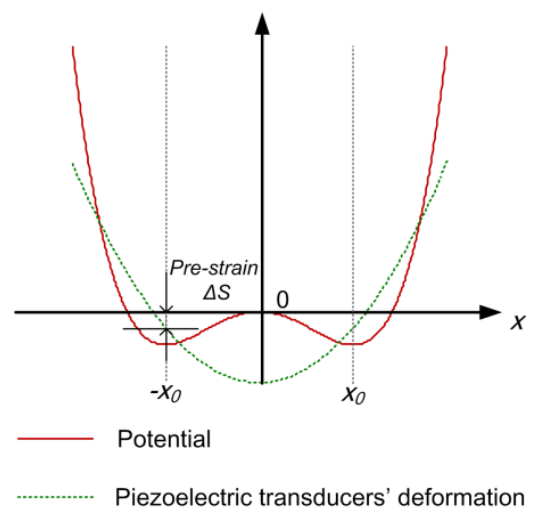

(b)

Figure 2. (a) Simplified sketch for the BSM generator modeling; (b) Potential and deformation curves. 
Considering the simplest case of a resistor $R$ as the electric load, eq.(9) can be further written as:

$$
\left\{\begin{array}{l}
\ddot{\bar{x}}-\frac{1}{2} \omega_{0}^{2} \bar{x}+\frac{1}{2} \frac{\omega_{0}^{2}}{\varepsilon^{2}} \bar{x}^{3}+2 \xi \omega_{0} \dot{\bar{x}}+\frac{k^{2}}{1-k^{2}} \frac{\omega_{0}^{2} \bar{x}}{\varepsilon} \bar{V}=\bar{\gamma}(t) \\
\omega_{r} \bar{V}-\frac{\bar{x} \dot{\bar{x}}}{\varepsilon}+\dot{\bar{V}}=0
\end{array}\right.
$$

where $\omega_{r}=1 / R / C_{0}$.

It is clear that the generator's performance is related to five normalized parameters: $\omega_{0}$ the characteristic frequency, $k^{2}$ the electromechanical coupling coefficient, $\xi$ the mechanical damping ratio, $\varepsilon$ the buckling level and $\omega_{r}$ the electric load. In this case, the normalized power is expressed as:

$$
\bar{P}=\frac{k^{2} \omega_{0}^{2} \omega_{r}}{1-k^{2}} \bar{V}^{2}
$$

Tab. 2. Normalized parameters and variables

\begin{tabular}{llll}
\hline Normalized parameters and variables & Symbol & Definition & Unit \\
\hline Buckling level & $\varepsilon$ & $x_{0} / L$ & \\
Electromechanical coupling coefficient & $k^{2}$ & $\alpha^{2} /\left(\alpha^{2}+K C_{0}\right)$ & \\
Mechanical damping coefficient & $\xi$ & $\mu /\left(2 \varepsilon K^{1 / 2} M^{1 / 2}\right)$ & \\
Characteristic angular frequency & $\omega_{0}$ & $\varepsilon K^{1 / 2} / M^{1 / 2}$ & $\mathrm{rad} \mathrm{s}^{-1}$ \\
Normalized displacement & $\bar{x}$ & $x / L$ & \\
Normalized excitation & $\bar{\gamma}$ & $\gamma / L$ & $\mathrm{~s}^{-2}$ \\
Normalized voltage & $\bar{V}$ & $V_{p} /\left(\alpha x_{0} / C_{0}\right)$ & \\
Normalized current & $\bar{I}$ & $I / \alpha x_{0}$ & $\mathrm{~s}^{-1}$ \\
Normalized power & $\bar{P}$ & $P /\left(M L^{2}\right)$ & $\mathrm{s}^{-3}$ \\
\hline
\end{tabular}

\section{Harmonic balance analysis and validation}

In this section, harmonic balance analysis is used to study the dynamic responses, especially for the desired inter-well motions under external harmonic excitations. Compared with traditional numerical and experimental investigation approaches for the nonlinear generators, the analytical method is more efficient on characterizing the dynamic properties of the BSM generator for different parameters' variations and providing important guiding information for design. It also provides a rapid and convenient solution for initial optimizations and evaluations.

It is well known that the periodic response for a harmonic excitation can be approximated by a truncated Fourier series. The number of the preserved orders determines the accuracy. Since it is shown in (Cohen et al., 2012) that the periodic motion of the bistable generator has a dominant fundamental frequency component and is similar to a sinusoidal signal, the motion solution of the BSM generator is assumed as:

$$
\left\{\begin{array}{l}
\bar{x}=c(t)+a \sin \omega t+b \cos \omega t \\
\dot{\bar{x}}=\dot{c}+(\dot{a}-b \omega) \sin \omega t+(\dot{b}+a \omega) \cos \omega t \\
\ddot{\bar{x}}=(2 \dot{a}-b \omega) \omega \cos \omega t-(\dot{b}+2 a \omega) \omega \sin \omega t
\end{array}\right.
$$

The amplitude of the motion is supposed to vary slowly so that the second or higher derivatives 
are neglected. $c(t)$ is the DC component of the intra-well motion and the value is zero for the inter-well motion as explicated (Stanton et al., 2012a).

\subsection{Intra-well motion}

When the generator vibrates around one of the two equilibrium positions, the displacement DC value can be considered as a constant, and it equals $\varepsilon$ in the case of small vibrations. Therefore, the deformation is assumed as approximately proportional to displacement. Then we estimate the piezoelectric voltage solution as:

$$
\left\{\begin{array}{l}
\bar{V}=p \sin \omega t+q \cos \omega t \\
\dot{\bar{V}}=(\dot{p}-q \omega) \sin \omega t+(\dot{q}+p \omega) \cos \omega t
\end{array}\right.
$$

with only the fundamental frequency kept.

Substituting eq. (13) and eq. (14) into eq. (11), removing the high-order harmonic terms and balancing the items for DC, $\sin \omega t$ and $\cos \omega t$ components, five equations are obtained:

$$
\left\{\begin{array}{l}
\frac{-c \omega_{0}^{2}}{2}+\frac{\left(3 a^{2}+3 b^{2}+2 c^{2}\right) c \omega_{0}^{2}}{4 \varepsilon^{2}}+\frac{(a p+b q) k^{2} \omega_{0}^{2}}{2 \varepsilon\left(1-k^{2}\right)}+2 \dot{c} \xi \omega_{0}=0 \\
-b \omega^{2}-\frac{b \omega_{0}^{2}}{2}+\frac{\left(3 a^{2}+3 b^{2}\right) b \omega_{0}^{2}}{8 \varepsilon^{2}}+\frac{3 b c^{2} \omega_{0}^{2}}{2 \varepsilon^{2}}+\frac{c k^{2} \omega_{0}^{2} q}{\varepsilon\left(1-k^{2}\right)}+2 a \xi \omega \omega_{0}+2 \dot{a} \omega+2 \dot{b} \xi \omega_{0}=\bar{\gamma} \\
-a \omega^{2}-\frac{a \omega_{0}^{2}}{2}+\frac{\left(3 a^{2}+3 b^{2}\right) a \omega_{0}^{2}}{8 \varepsilon^{2}}+\frac{3 a c^{2} \omega_{0}^{2}}{2 \varepsilon^{2}}+\frac{c k^{2} \omega_{0}^{2} p}{\varepsilon\left(1-k^{2}\right)}-2 b \xi \omega \omega_{0}-2 \dot{b} \omega+2 \dot{a} \xi \omega_{0}=0 \\
\frac{a c \omega}{\varepsilon}-p \omega-q \omega_{r}+\frac{\dot{b} c}{\varepsilon}+\frac{b \dot{c}}{\varepsilon}-\dot{q}=0 \\
\frac{b c \omega}{\varepsilon}-q \omega+p \omega_{r}-\frac{\dot{a} c}{\varepsilon}-\frac{a \dot{c}}{\varepsilon}+\dot{p}=0
\end{array}\right.
$$

In a steady state operation, eq. (15) becomes:

$$
\left\{\begin{array}{l}
\frac{-c \omega_{0}^{2}}{2}+\frac{\left(3 a^{2}+3 b^{2}+2 c^{2}\right) c \omega_{0}^{2}}{4 \varepsilon^{2}}+\frac{(a p+b q) k^{2} \omega_{0}^{2}}{2 \varepsilon\left(1-k^{2}\right)}=0 \\
-b \omega^{2}-\frac{b \omega_{0}^{2}}{2}+\frac{\left(3 a^{2}+3 b^{2}\right) b \omega_{0}^{2}}{8 \varepsilon^{2}}+\frac{3 b c^{2} \omega_{0}^{2}}{2 \varepsilon^{2}}+\frac{c k^{2} \omega_{0}^{2} q}{\varepsilon\left(1-k^{2}\right)}+2 a \xi \omega \omega_{0}=\bar{\gamma} \\
-a \omega^{2}-\frac{a \omega_{0}^{2}}{2}+\frac{\left(3 a^{2}+3 b^{2}\right) a \omega_{0}^{2}}{8 \varepsilon^{2}}+\frac{3 a c^{2} \omega_{0}^{2}}{2 \varepsilon^{2}}+\frac{c k^{2} \omega_{0}^{2} p}{\varepsilon\left(1-k^{2}\right)}-2 b \xi \omega \omega_{0}=0 \\
\frac{a c \omega}{\varepsilon}-p \omega-q \omega_{r}=0 \\
\frac{b c \omega}{\varepsilon}-q \omega+p \omega_{r}=0
\end{array}\right.
$$

With $r=\left(a^{2}+b^{2}\right)^{1 / 2}$ and $r_{v}=\left(p^{2}+q^{2}\right)^{1 / 2}$ standing for the amplitude of the displacement and the voltage respectively, the equation group (16) can be simplified as: 


$$
\left\{\begin{array}{l}
c=\left(\varepsilon^{2}-\frac{3}{2} r^{2}-\frac{k^{2} \omega^{2} r^{2}}{\left(1-k^{2}\right)\left(\omega^{2}+\omega_{r}^{2}\right)}\right)^{1 / 2} \\
r=\frac{\bar{\gamma}}{\left(M_{a}^{2}+M_{b}^{2}\right)^{1 / 2}} \\
r_{v}=\frac{c r \omega}{\varepsilon\left(\omega^{2}+\omega_{r}^{2}\right)^{1 / 2}}
\end{array}\right.
$$

where

$$
\left\{\begin{array}{l}
M_{a}=-\omega^{2}-\frac{\omega_{0}^{2}}{2}+\frac{3 r^{2} \omega_{0}{ }^{2}}{8 \varepsilon^{2}}+\frac{3 c^{2} \omega_{0}{ }^{2}}{2 \varepsilon^{2}}+\frac{c^{2} k^{2} \omega_{0}{ }^{2} \omega^{2}}{\varepsilon^{2}\left(1-k^{2}\right)\left(\omega^{2}+\omega_{r}{ }^{2}\right)} \\
M_{b}=\frac{c^{2} k^{2} \omega_{0}{ }^{2} \omega \omega_{r}}{\varepsilon^{2}\left(1-k^{2}\right)\left(\omega^{2}+\omega_{r}{ }^{2}\right)}+2 \xi \omega \omega_{0}
\end{array}\right.
$$

The frequency response of the BSM generator is finally determined by finding the real roots of eq. (17).

Figure 3 depicts the intra-well displacements responses for different damping cases. The thick lines represent stable solutions while the thin lines are unstable solutions. The stability of each solution is figured out using the perturbation method (Nayfeh and Mook, 2008). For a given solution $s\left(a_{0} ; b_{0}\right.$; $\left.c_{0} ; p_{0} ; q_{0}\right)$, a small perturbation $\Delta s(\Delta a ; \Delta b ; \Delta c ; \Delta p ; \Delta q)$ is applied so that we can develop $s+\Delta s$. Substituting this perturbed solution into eq. (16) and linearizing it for small $\Delta s$, we obtain a new differential equation set for the new variable $\Delta s$. The stability of the solution $s$ is determined along with the eigenvalue of the coefficient matrix: stable if all the eigenvalues have negative real parts; unstable if there are any positive real parts. This stability determination is also applied for the following inter-well solution. As seen in the figure 3, the BSM generator behaves as a softening Duffing oscillator while the nonlinear effect decreases as the damping coefficient increases.

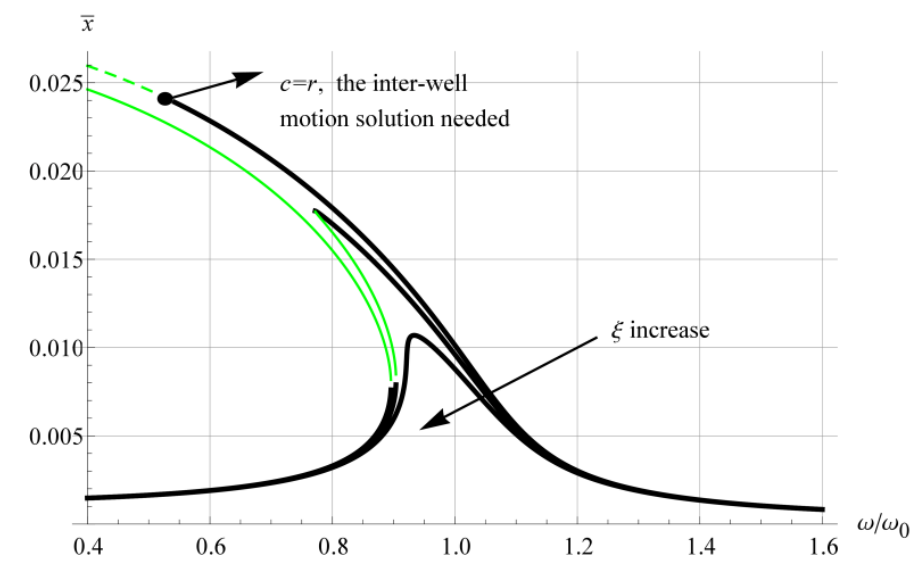

Figure 3. Intra-well displacement responses for different damping coefficients $\left(\bar{\gamma}=15.2, \varepsilon=0.0379, \omega_{0}=110, \omega_{r}=\omega_{0}, k^{2}=0.07, \quad \xi=\{0.0156,0.03,0.045\}\right)$. Thick lines, stable solutions; Thin lines, unstable solutions.

The DC component $c$ for the intra-well motion is related to the amplitude because the system in 
this case is not symmetrical around the equilibrium position. As the amplitude increases, $c$ will drift from $\varepsilon$ towards the position $\bar{x}=0$. The relationship between $c$ and $r$ is pictured in figure 4 . As the displacement amplitude $r$ goes to $0.8 \varepsilon, c$ gets close to the zero position. However, it is worthy of note that the amplitude $r$ for the inter-well motion needs to satisfy $r<c$. Otherwise, the motion of the generator has crossed the potential saddle $(c-r<0)$ and should be analyzed as the inter-well motion case. The converging point of the red thin line and the thick line in figure 4 indicates this transition point. The intra-well solutions are valid at the left side of the critical point. When the amplitude goes to the right side, the inter-well solutions are applicable. An example of the transition from the intra-well motion to the inter-well motion is demonstrated in figure 3 for $\xi=0.0156$. The amplitude responses that are higher than the indicated critical point in the figure are no longer valid as stable solutions.

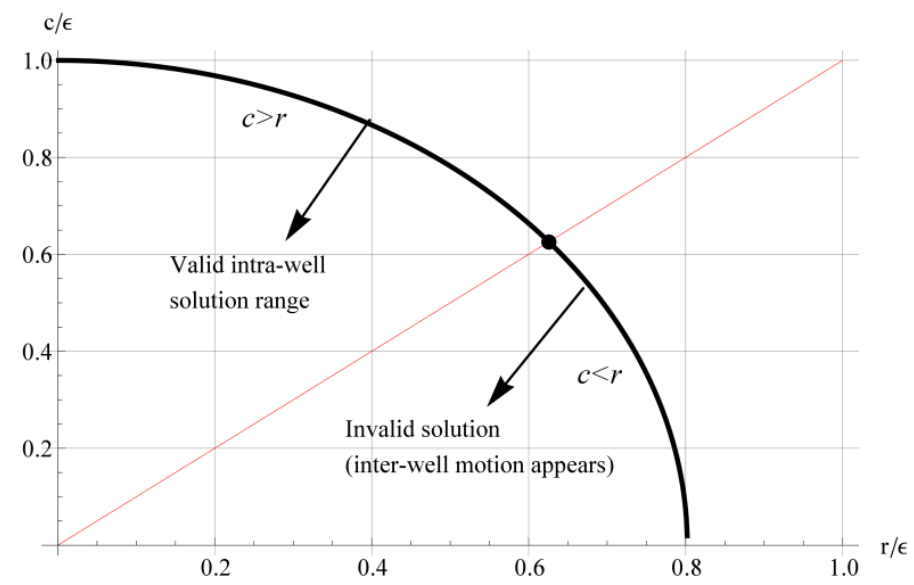

Figure 4. DC component $c$ versus the displacement response amplitude $r\left(\varepsilon=0.0379, \omega_{r}=\omega_{0}=110\right.$, $\left.k^{2}=0.07, \xi=0.0156\right)$.

\subsection{Inter-well motion}

Since the most desired properties come from the inter-well motion of the bistable generator, main efforts go to the solutions in this case. With the same assumed displacement solution as eq. (13), the voltage solution for the inter-well motions is a little different. Since the deformation of the piezoelectric components is symmetric about the position $x=0$ as figure 2 (b), the same is applicable to the piezoelectric voltage. Then in a period of a harmonic inter-well motion, the piezoelectric voltage has twice the identical waveform for the $x>0$ and $x<0$ range. It means the voltage frequency is two times the displacement frequency. Moreover, it is observed in the electric equation in eq. (11) that the voltage is proportional to the product of the displacement and the velocity. As a result, the frequency is doubled according to the trigonometric formula. Then we have:

$$
\left\{\begin{array}{l}
\bar{V}=p \sin 2 \omega t+q \cos 2 \omega t \\
\dot{\bar{V}}=(\dot{p}-2 q \omega) \sin 2 \omega t+(\dot{q}+2 p \omega) \cos 2 \omega t
\end{array}\right.
$$

With the same operations as for the intra-well motion, the equation group for solving the steady states is: 


$$
\left\{\begin{array}{l}
c=0 \\
-b \omega^{2}-\frac{b \omega_{0}^{2}}{2}+\frac{\left(3 a^{2}+3 b^{2}\right) b \omega_{0}^{2}}{8 \varepsilon^{2}}+\frac{3 b c^{2} \omega_{0}^{2}}{2 \varepsilon^{2}}+\frac{k^{2} \omega_{0}^{2}(a p+b q)}{2 \varepsilon\left(1-k^{2}\right)}+2 a \xi \omega \omega_{0}=\bar{\gamma} \\
-a \omega^{2}-\frac{a \omega_{0}^{2}}{2}+\frac{\left(3 a^{2}+3 b^{2}\right) a \omega_{0}^{2}}{8 \varepsilon^{2}}+\frac{3 a c^{2} \omega_{0}^{2}}{2 \varepsilon^{2}}+\frac{k^{2} \omega_{0}^{2}(a q+b p)}{2 \varepsilon\left(1-k^{2}\right)}-2 b \xi \omega \omega_{0}=0 \\
\frac{a b \omega}{\varepsilon}-2 p \omega-q \omega_{r}=0 \\
\frac{\left(a^{2}-b^{2}\right) \omega}{2 \varepsilon}+2 q \omega-p \omega_{r}=0
\end{array}\right.
$$

After the simplification, we have the final equations:

$$
\left\{\begin{array}{l}
c=0 \\
r=\frac{\bar{\gamma}}{\left(M_{p}{ }^{2}+M_{q}^{2}\right)^{1 / 2}} \\
r v=\frac{r^{2} \omega}{2 \varepsilon\left(4 \omega^{2}+\omega_{r}{ }^{2}\right)^{1 / 2}}
\end{array}\right.
$$

where

$$
\left\{\begin{array}{l}
M_{p}=-\omega^{2}-\frac{\omega_{0}^{2}}{2}+\frac{3 r^{2} \omega_{0}^{2}}{8 \varepsilon^{2}}+\frac{3 c^{2} \omega_{0}{ }^{2}}{2 \varepsilon^{2}}+\frac{r^{2} k^{2} \omega_{0}{ }^{2} \omega^{2}}{2 \varepsilon^{2}\left(1-k^{2}\right)\left(4 \omega^{2}+\omega_{r}{ }^{2}\right)} \\
M_{q}=\frac{r^{2} k^{2} \omega_{0}{ }^{2} \omega \omega_{r}}{4 \varepsilon^{2}\left(1-k^{2}\right)\left(4 \omega^{2}+\omega_{r}{ }^{2}\right)}+2 \xi \omega \omega_{0}
\end{array}\right.
$$

Figure 5 shows an inter-well frequency response for a given BSM generator by solving eq. (21). The top thick line represents the stable inter-well solution while the two red thin lines are the unstable solutions. Since eq. (21) only accounts for the high-energy inter-well orbits, it is necessary to use the eq. (17) to obtain the low-energy intra-well orbits as shown by the two thick lines (stable) below and the green thin line (unstable) for the purpose of completed solutions. It is clear that for the same excitation, the inter-well motion has a much better response than the intra-well case, thus (resulting in) more power to be harvested. The dashed lines with arrows indicate the jump positions between the high-energy orbit and the low-energy orbit. The jump-up arrow represents the harvester transiting from the low-energy orbit to the high-energy one for the reverse sweep while the jump-down arrow is the position from the high-energy orbit to the low-energy one for the forward sweep. These jump positions are obtained through the stability boundary of the solution as shown in figure 5. The special hysteresis phenomena of the nonlinear system response are easily inferred from the jump indications. 


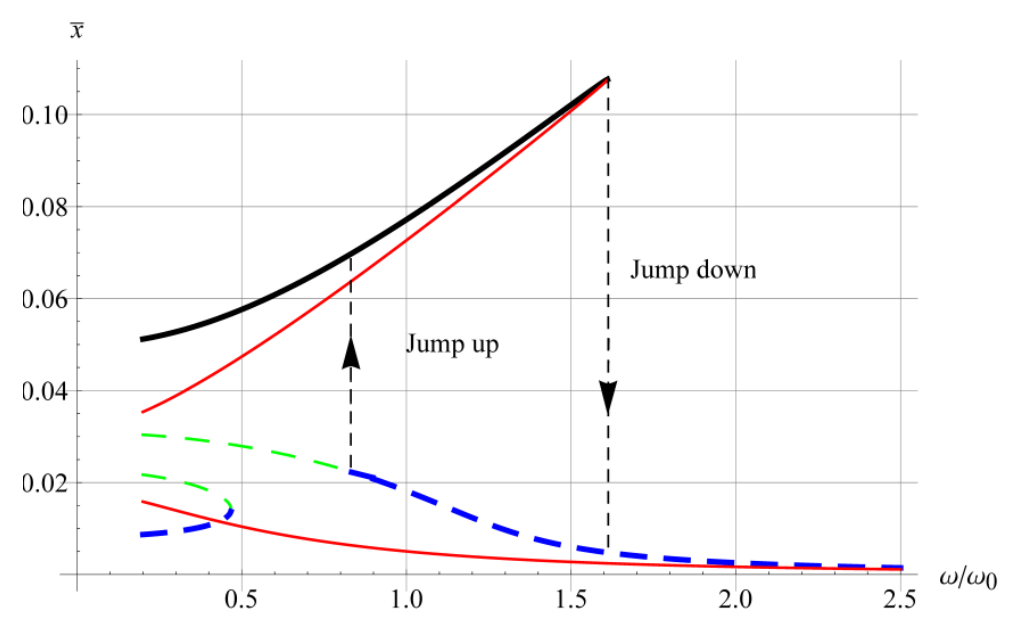

Figure 5. Inter-well (solid lines) and intra-well (dashed lines) displacement responses of a BSM generator $\left(\bar{\gamma}=91, \varepsilon=0.0379, \omega_{0}=110, \omega_{r}=\omega_{0}, k^{2}=0.07, \xi=0.0156\right)$. Thick lines, stable solutions; Thin lines, unstable solutions.

\subsection{Experimental validation}

In order to validate the model obtained with harmonic balance analysis, the analytical solution is compared with the experimental results for a BSM generator prototype (Liu et al., 2013a) with the parameters listed in Tab.3.

Tab. 3. Parameters for the BSM generator

\begin{tabular}{ccccccccc}
\hline$M(\mathrm{~g})$ & $L(\mathrm{~mm})$ & $K(\mathrm{~N} / \mathrm{m})$ & $x_{0}(\mathrm{~mm})$ & $C_{0}(\mu F)$ & $\alpha(\mathrm{N} / \mathrm{l})$ & $\mu(N \bullet \mathrm{s} / \mathrm{m})$ & $R(K \Omega)$ & $\gamma\left(\mathrm{m} / \mathrm{s}^{2}\right)$ \\
\hline 45.8 & 33 & $3.9 \mathrm{e} 5$ & 1.25 & 2.3 & 0.26 & 0.16 & 3.84 & 3 \\
\hline$\varepsilon$ & $\omega_{0}(\mathrm{rad} / \mathrm{s})$ & $k^{2}$ & $\xi$ & $\omega_{r}(\mathrm{rad} / \mathrm{s})$ & $\bar{\gamma}\left(\mathrm{s}^{-2}\right)$ & & & \\
\hline 0.0379 & 110.5 & 0.0684 & 0.016 & 113 & 91 & & & \\
\hline
\end{tabular}

The prototype includes four flexible hinges made from machined PTFE material and two APA120S from Cedrat-Technologies actuator used as the piezoelectric components (Figure 1). A tuning screw is utilized to adjust the buckling level $\varepsilon$. Fixed on a horizontal shaker table, the prototype is excited using a chirp acceleration of amplitude $3 \mathrm{~m} / \mathrm{s}^{2}$ from $7 \mathrm{~Hz}-42 \mathrm{~Hz}$ with a rate of $0.025 \mathrm{~Hz} / \mathrm{s}$ which is slow enough to obtain the steady state responses. A Matlab ${ }^{\odot}$ program is used to produce the desired excitation signals and communicate with the real-time control board (RTI1103, dSpace ${ }^{\odot}$ ). The shaker (2075E-HT, The Modal Shop ${ }^{\odot}$ ) is driven through a PI feedback loop using an accelerometer $\left(\mathrm{M} 352 \mathrm{C} 68, \mathrm{PCB}^{\odot}\right)$ to ensure constant acceleration amplitude. A differential optical vibrometer $\left(\mathrm{OFV}-552\right.$ and OFV-5000, Polytec $^{\circ}$ ) allows the displacement of the central mass to be measured. Finally, a load resistance is selected according to the classical strategy for linear harvesters as follows:

$$
R=\frac{1}{C_{0} \omega_{0}}
$$

Due to the resistor box value limitation, a resistor $3.84 \mathrm{k} \Omega$ is used in reality. Then we have $\omega_{r}=113(\approx$ $\left.\omega_{0}\right)$ in this case. 


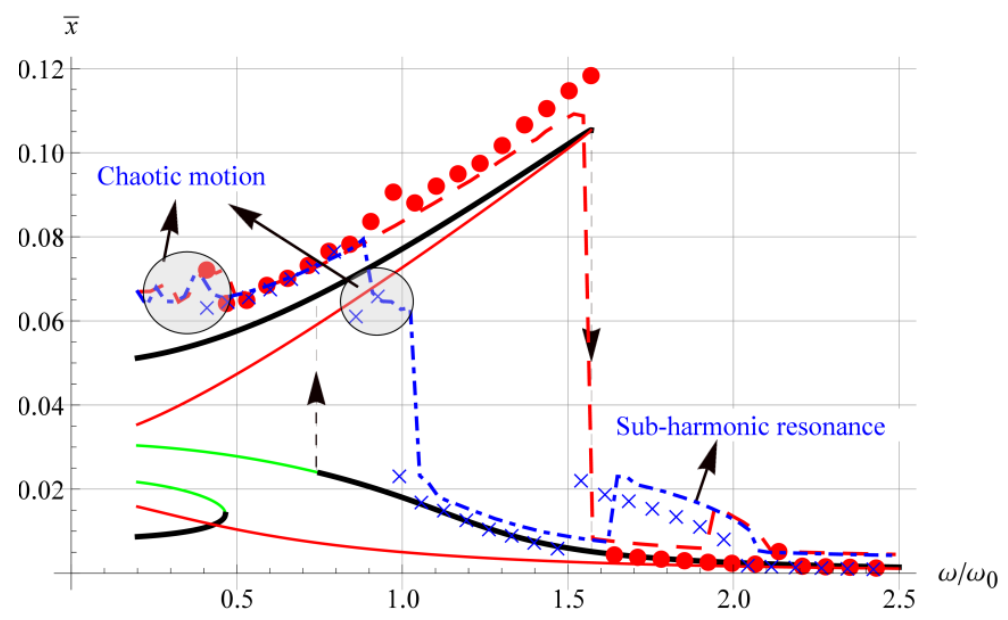

(a)

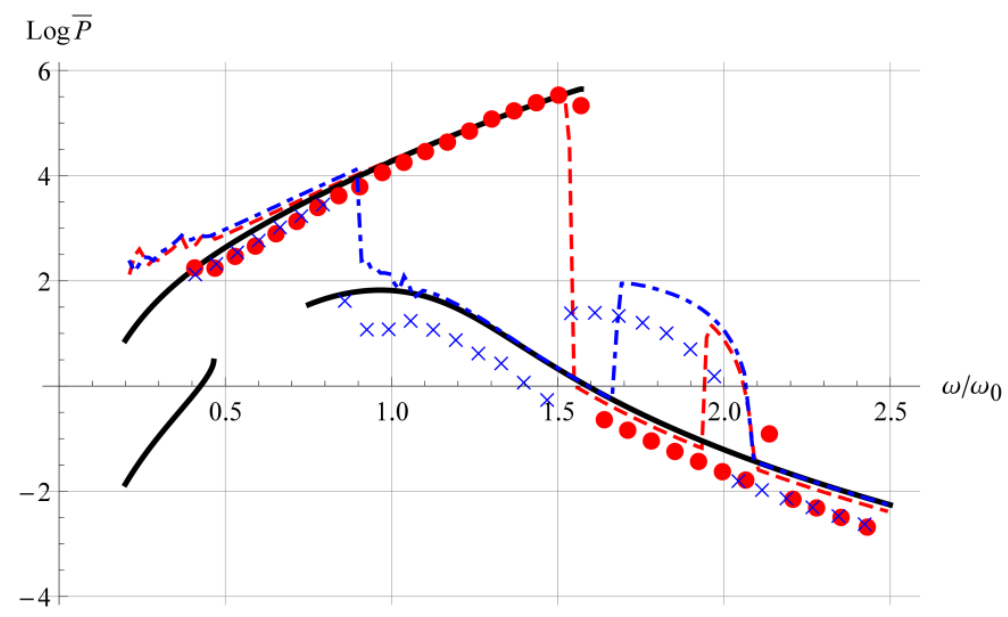

(b)

Figure 6. Analytical results vs experimental results for a BSM prototype: (a) Displacement; (b) Power. Solid circles: experimental results of forward sweep; Crosses: experimental results of reverse sweep; Thick lines: stable analytical solutions; Thin lines: unstable analytical solutions; Dashed lines: numerical results of forward sweep; Dash-dot lines: numerical results of reverse sweep.

Figure 6 presents the displacement and power responses with three different approaches: analytical, numerical and experimental. The solid circles represent the experimental forward sweep response and the crosses the reverse sweep response. The thick lines are stable solutions while the thin lines are unstable solutions calculated from the analytical model. Numerical results for the forward sweep and the reverse sweep are plotted with dashed and dash-dot lines respectively. As we can see, the experimental results are very close to the analytical and numerical ones. However, we can observe relatively large deviation at some positions between the analytical and experimental results indicated by the texts. It is due to the chaotic motions or the super-harmonic resonance (Nayfeh AH and Mook DT, 2008) of the BSM generator.

It is worthy of note that this chaotic motion cannot be analyzed with the harmonic balance method or other analytical methods with periodic motion assumptions. Although Melnikov approach presents a common way of predicting chaos, it is only usable in weakly excited systems (Stanton et al. 
2012b) with preliminary conclusions while the happening of chaos is still difficult to foresee accurately. Besides, since the harmonic balance method only considers the fundamental frequency component, the super-harmonic resonance is not included in the model either. However, as the chaotic motion and the super- and sub- harmonic resonances are infrequent events, it is not so relevant when considering the power performance (only the stable solutions) as shown in figure 6 (b). Thus, further efforts for detailing these two phenomena are beyond the scope of this article.

By inspecting figure 6, better agreements between the experimental results and the numerical results are found, especially for the chaos and super-harmonic resonance observed in the simulation as well. However, since the solutions of the bistable generator is strongly dependent on the initial condition (Erturk and Inman, 2011; Nayfeh AH and Mook DT, 2008), the numerical simulation can only give the specific solution corresponding to a determined initial status as the experimental approach, while losing the completeness of the solution space. As shown in figure 6, the intra-well solution at the low frequency side is not visible. Moreover, due to the high time cost for the numerical simulation, it is difficult to perform complete parameter optimization study as the analytical approach.

Despite of the small discrepancies, the good correlation between experiment and theory confirms that the analytical model is fully capable of predicting the performance of the BSM generator. Thus, we can conclude that it can be an efficient tool in the design and optimization, especially at the early stage.

\section{Discussion}

Compared with commonly used numerical methods, the analytical model is able to provide simulation results in a much quicker way. Moreover, the whole solution space of the dynamic system is provided by the analytical method whereas numerical simulation results are related to the chosen initial conditions. As a result, the analytical model presents more details of the system, which helps to understand the generator's behavior. In this section, the influence of the five critical parameters $\left(\xi, \omega_{r}, k^{2}, \varepsilon, \omega_{0}\right)$ on the generator's performance is studied with the analytical model. As a result, the optimization of the BSM generator is provided with theoretical support. As the basis for further discussion, the generator is assumed to have the same inertial mass $M$ and feature size $L$ as well as identical excitation case considered $\left(\bar{\gamma}=91 \mathrm{~s}^{-2}, 22 \mathrm{rad}\right.$ $\left.\mathrm{s}^{-1}-275 \mathrm{rad} \mathrm{s}^{-1}\right)$. Since the high-energy inter-well response is sought, the discussion is mainly focused on this case.

\subsection{Damping ratio $\xi$}

Figure 7 shows the inter-well responses for the increasing damping coefficient that might be from the mechanical part or the electric part or both. As can be seen, the BSM generator jumps from the high-energy orbit to the low-energy orbit earlier for higher damping cases as expected (Stanton et al., 2012a). Nevertheless, the influence of the increasing damping on the low-energy and the high-energy orbits is not so obvious below the jump frequencies. The responses at these positions are almost identical, similar to the case of vibration with constant displacement amplitude. The jump locations from low-energy to high-energy orbits are not affected either. It implies that, in these cases, the generator is not sensitive to the damping. As a result, it is possible to extract more energy with the circuits in these situations. However, from a global perspective, it would be better to aim at lower damping systems to have a wider band and higher power output. 


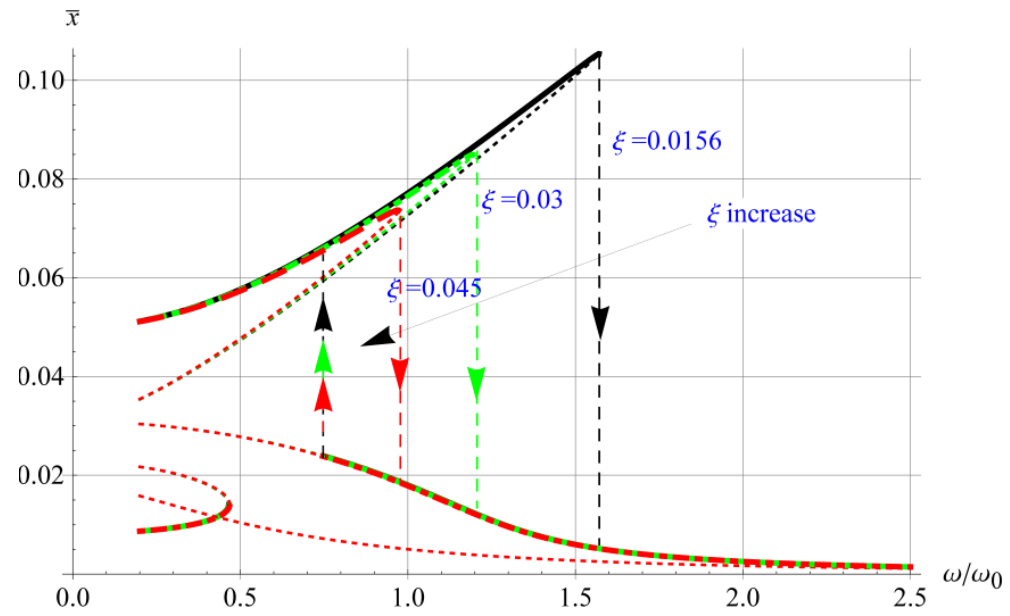

Figure 7. Inter-well displacement responses for different damping ratios. $\left(\bar{\gamma}=91 \mathrm{~s}^{-2}, \varepsilon=0.0379, \omega_{0}=110\right.$ rad s$\left.s^{-1}, \omega_{r}=\omega_{0}, k^{2}=0.07, \xi=\{0.0156,0.03,0.045\}\right)$. Thick lines: stable solutions; Dotted lines: unstable solutions; Arrows: the jumps between the high-energy stable orbits to low-energy stable orbits.

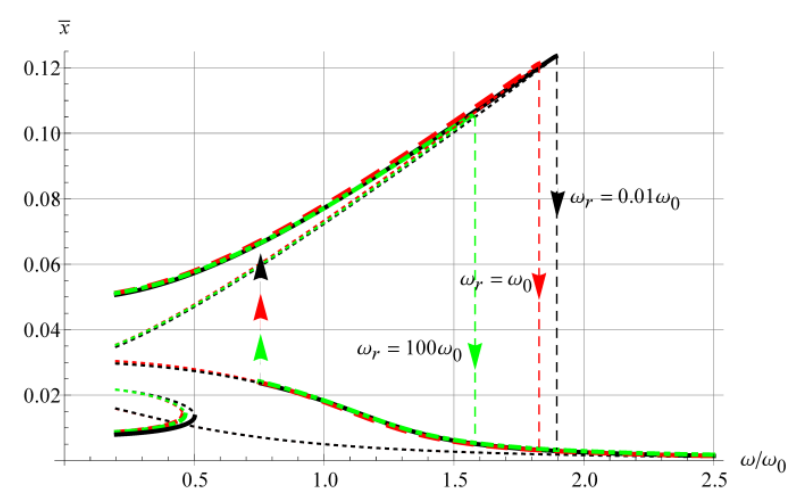

(a)

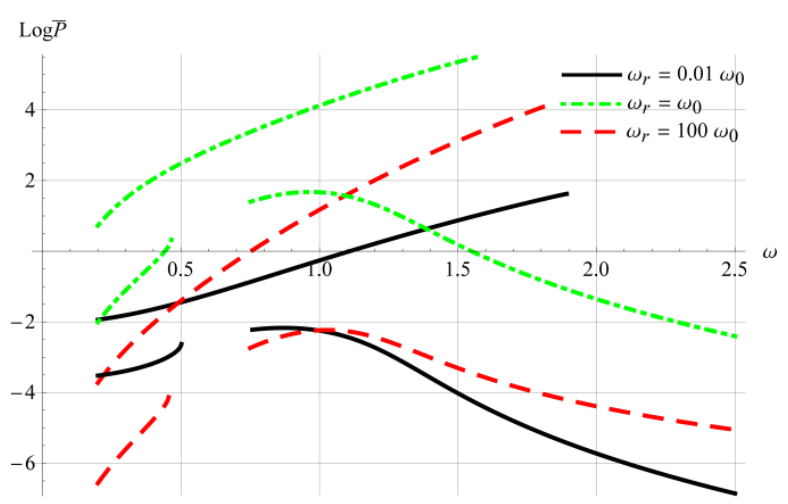

(b)

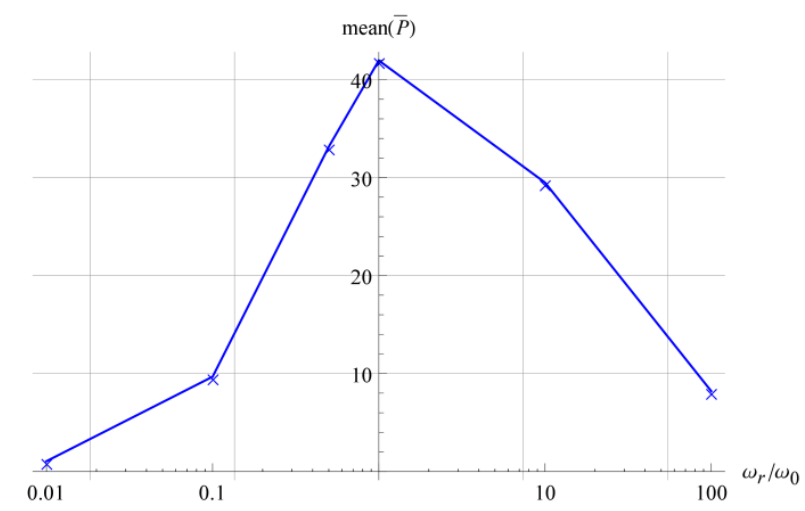

(c)

Figure8 (a) Inter-well displacement responses for different resistor values; (b) Corresponding power responses. (c) Average power of inter-well motions versus different load values over the concerned

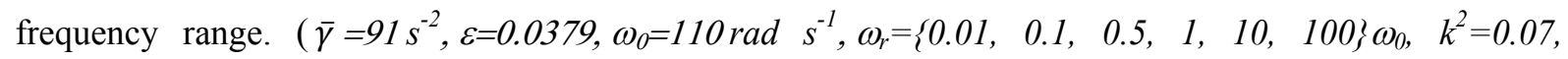
$\xi=0.0156$ ). 


\subsection{Electric load related pulsation $\omega_{r}$}

The electric load related pulsation $\omega_{r}=1 /\left(R C_{0}\right)$ represents the load. It has strong relationship to the application and influences the harvested power and the dynamic displacement responses eventually. Figure 8 (a) and (b) shows the inter-well displacement responses and the corresponding power for different resistor values (for fixed $C_{0}$ ). For the power, only the meaningful stable solutions are considered. It is interesting to find out that the inter-well motion range first decreases then increases with $\omega_{r}\left(\omega_{r}=0.01 \omega_{0} \rightarrow \omega_{r}=\omega_{0}\right)$. The jump position from the high to the low energy orbits moves from high frequencies indicated by the green arrow to the low frequencies shown by the black arrow. However, we can see the harvested power enhanced by comparing the power curves for these two different $\omega_{r}$ cases. As $\omega_{r}$ keeps on increasing until $100 \omega_{0}$, the jump-down position shifts back to a much higher frequency while the power decreases sharply at the same time. When looking back at the influence of the damping on the system responses, it seems that the power dissipated at the load acts as a specific electrical damping for the generator motion as pointed out in this article (Liu et al., 2013b).

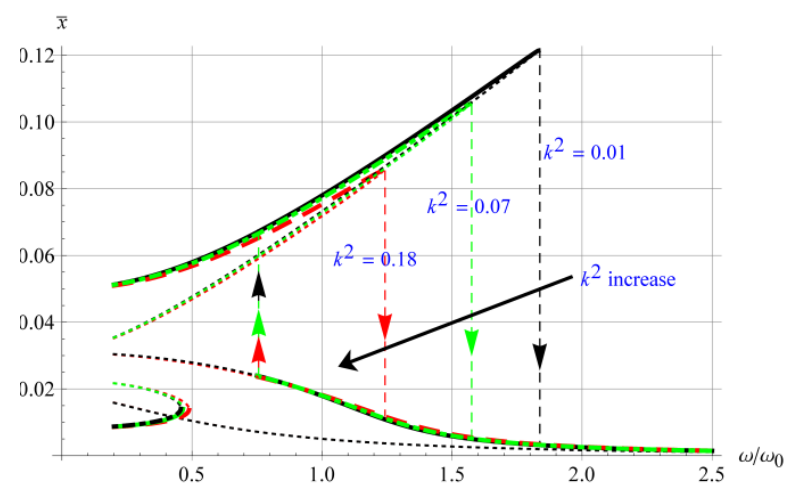

(a)

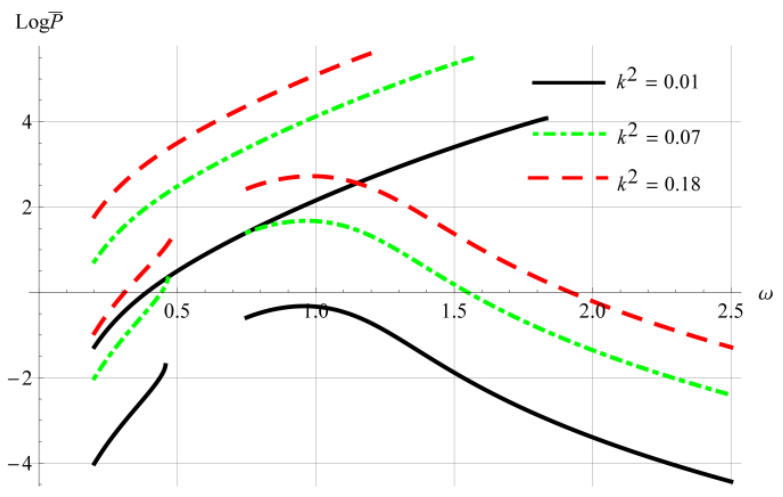

(b)

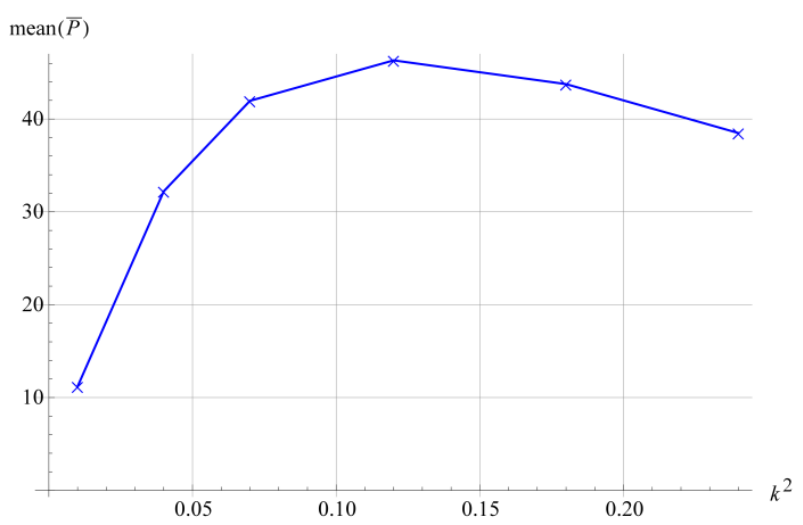

(c)

Figure9 (a) Inter-well displacement responses for different coupling levels; (b) Corresponding power responses. (c) Average normalized power of inter-well motions over the concerned frequency range versus coupling levels. $\left(\bar{\gamma}=91 \mathrm{~s}^{-2}, \varepsilon=0.0379, \omega_{0}=110 \mathrm{rad} \mathrm{s}^{-1}, \omega_{r}=\omega_{0}, k^{2}=\{0.01,0.035,0.07,0.18,0.24\}, \xi=0.0156\right)$.

Moreover, from the power response trends for three different load conditions, one can infer that there is an optimal value of the load resistor to get the maximum total harvested power over a specific frequency range. The trade-off between the harvested energy and the operation bandwidth has to be considered. To get a clear relationship between the harvested power and the load value, the average normalized power of the inter-well motion over the concerned frequency range 
$\left(\omega_{1}=0.2 \omega_{0} \rightarrow \omega_{2}=2.5 \omega_{0}\right)$

$$
\operatorname{mean}(\bar{P})=\frac{1}{\omega_{2}-\omega_{1}} \int_{\omega_{1}}^{\omega_{2}} \bar{P} d \omega
$$

is calculated for five load cases $\omega_{r}=\{0.01,0.1,1,10,100\} \omega_{0}$. A notable issue to be pointed out is that only the high-energy orbit considered. For the frequency range without inter-well motions, $\bar{P}$ is considered as zero for the power contribution of the intra-well motions in these cases is negligible. It is seen in figure 8 (c) that an optimal load value exists around $\omega_{r}=\omega_{0}$. As $\omega_{r}$ changes from $0.5 \omega_{0} \rightarrow 10 \omega_{0}$, the power stays above $70 \%$ of the maximum value. Therefore, the availability of a satisfying power level for a considerable load range is an obvious advantage of the bistable generator.

\subsection{Coupling level $k^{2}$}

Figure 9 (a) and (b) pictures the displacement responses and the power responses respectively for three different coupling levels. As we can see, the increase of the coupling level leads to a decrease of the inter-well motion range and an increase of the available power for the frequency points where the inter-well motions appear in each case. The shrink of the inter-well motion is due to the enlarged damping brought by the increased harvested energy and piezoelectric force. Figure 9 (c) presents the average normalized power of the inter-well motions defined in eq. (24) for more different coupling levels.

It is shown in figure 9 (c) that the power first rapidly increases with $k^{2}$ when its value is small. Then the power increases slowly until reaching its maximum value. Finally, it begins to decrease very slowly with increasing coupling. As the coupling level keeps on increasing, it brings more damping in the form of harvested energy so that the displacement amplitude decreases obviously. As a result, the power increase slows down when it approaches the apex. As the coupling continually rises to a nonrealistic value, the dynamic response of the generator is further suppressed. As a result, the power response will decrease except an alternative optimal load value selected. This trend is very similar to the relationship between the load and the coupling level in linear cases (Arroyo et al., 2012).

\subsection{Buckling level $\varepsilon$ and characteristic frequency $\omega_{0}$}

Considering the normalized equation (11), we can deduce the normalized potential expression with first integration:

$$
\bar{U}=-\frac{1}{4} \omega_{0}^{2} \bar{x}^{2}+\frac{\omega_{0}^{2} \bar{x}^{4}}{8 \varepsilon^{2}}
$$

In order to have the inter-well motions, the BSM generator has to cross the potential barrier at the center:

$$
\Delta \bar{U}=-\frac{1}{8} \omega_{0}^{2} \varepsilon^{2}
$$

These two equations suggest that the static properties of the BSM structure are driven by $\varepsilon$ and $\omega_{0}$.

The buckling level $\varepsilon$ is an extra parameter brought by the bistable architecture compared with linear generators. It is relevant to the nonlinearity level and induces important impact on the BSM 
generator's performance. Figure 10 (a) shows the displacement responses of the BSM generators with three different buckling levels. As $\varepsilon$ increases, the inter-well motion range decreases while the displacement amplitude of the inter-well motion increases. It is interesting to notice three stable solutions in a small frequency range for the highest $\varepsilon$ case. The middle stable solution has two possible jump directions as shown by the red arrows: to the upper high-energy inter-well orbit or to the bottom low-energy intra-well orbit.

As pictured in figure 10 (b), the generator has the largest power but the worst inter-well motion range in the highest $\varepsilon$ case while the generator has the widest inter-motion range but the drastically decreased power amplitude in the smallest $\varepsilon$ case; in the intermediate $\varepsilon$ case, the inter-well motion range and the power amplitude are both acceptable. It suggests that the trade-off between bandwidth and power has to be sought for the best performance as well. Still using the average normalized power over the whole excitation frequency range as the figure of merit, the performance for different buckling levels is studied in figure 10 (c). Clearly, an optimal buckling level is then found. Indeed, high buckling levels mean high potential barriers that restrict the available inter-well motion. Low buckling levels make the generators similar to mono-stable generators without the advantages of power enhancement due to the snap-through behavior, especially over the low frequency range. Therefore, a proper buckling level has to be determined according to the excitation while the analytical model provides a good tool for seeking this optimal value.

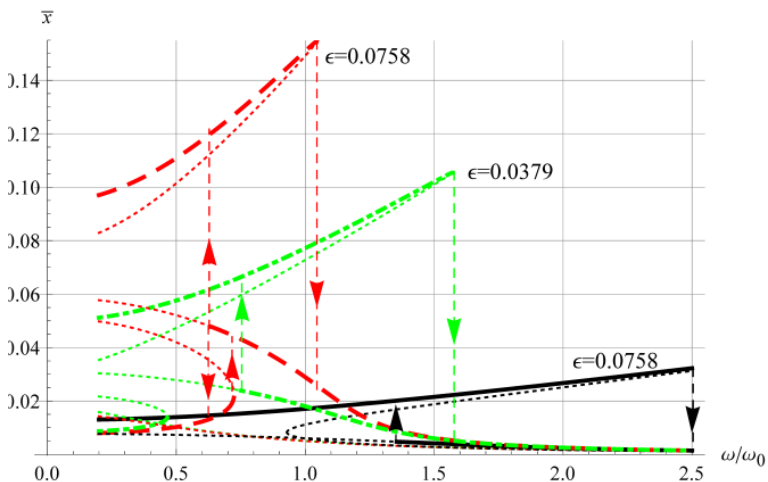

(a)

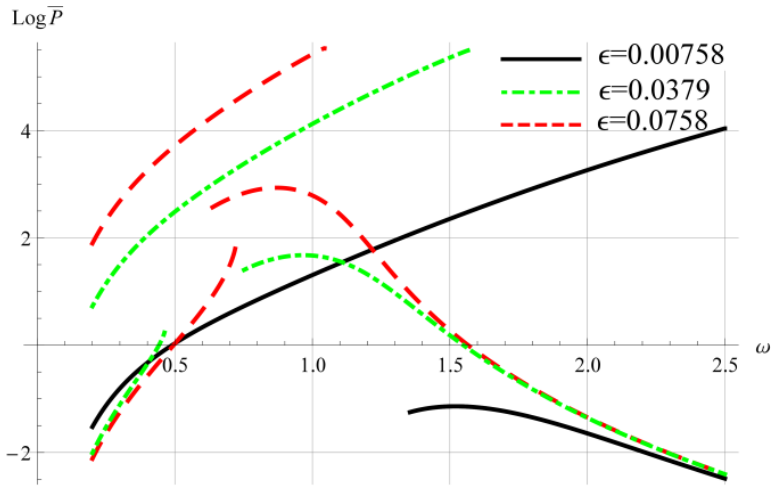

(b)

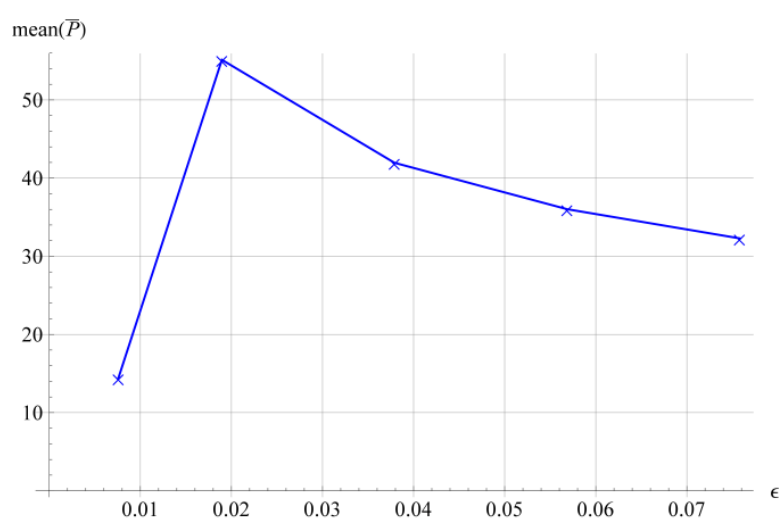

(c)

Figure 10. (a) Inter-well displacement responses for different buckling levels; (b) Corresponding power responses. (c) Average normalized power of inter-well motions over the concerned frequency range versus buckling levels. $\left(\bar{\gamma}=91 \mathrm{~s}^{-2}, \omega_{0}=110 \mathrm{rad} \mathrm{s}^{-1}, \omega_{r}=\omega_{0}, k^{2}=\{0.01,0.035,0.07,0.18,0.24\}, \xi=0.0156\right)$. 
Concerning the critical characteristic frequency $\omega_{0}$ of the BSM generator, the results obtained by the analytical model are presented in figure 11 (a) for different cases. As $\omega_{0}$ increases, the inter-well motion range and the displacement amplitude get worse at the same time. However, the power amplitude in the inter-well motion range is not impacted much for all three cases as indicated in figure 11 (b). In cooperation with the power performance in figure 10 (b) for different $\varepsilon$, it seems that the power amplitude is more related to the buckling level $\varepsilon$, less influenced by $\omega_{0}$. That is somehow contrary to the normal sense that the power amplitude is almost equally affected by $\varepsilon$ and $\omega_{0}$ that determine the potential shape of the BSM generator together.

However, considering that small $\omega_{0}$ induces lower potential barrier, the inter-well motion range is extended. Then the total energy harvested in the concerned frequency range is enlarged. It is validated by the average normalized power for different $\omega_{0}$ values in figure 11 (c). The performance of the BSM generator declines with higher $\omega_{0}$ values.

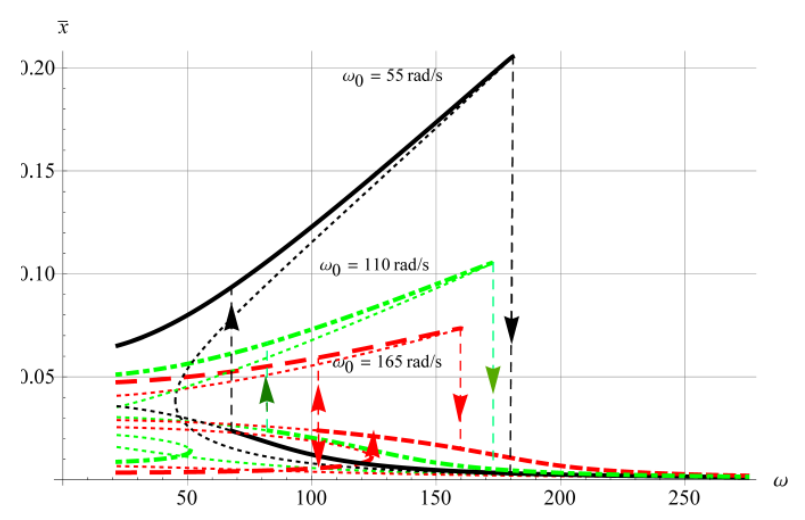

(a)

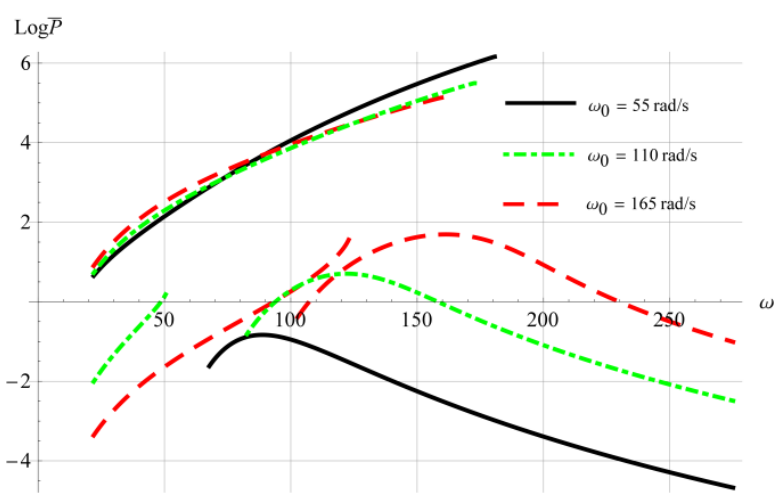

(b)

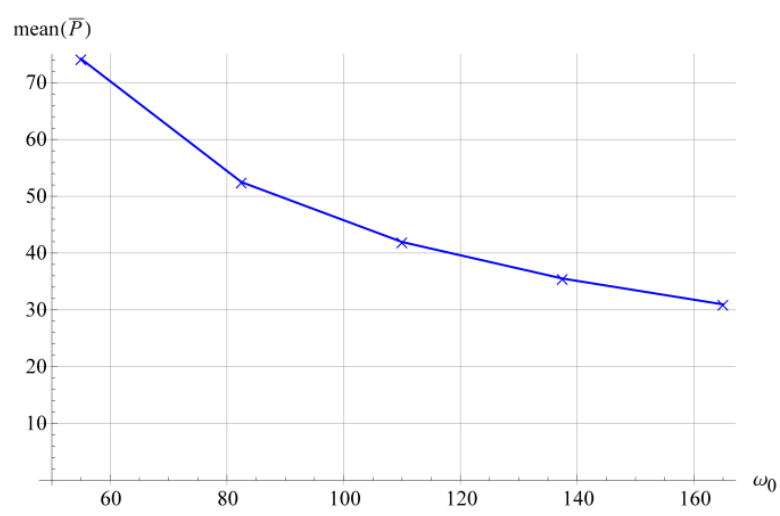

(c)

Figure 11. (a) Inter-well displacement responses for different characteristic frequencies; (b) Corresponding power responses. (c) Average normalized power of inter-well motions versus $\omega_{0}$. $\left(\bar{\gamma}=91 \mathrm{~s}^{-2}, \varepsilon=0.0379, \omega_{0}=\{55,82.5110,137.5,165\} \mathrm{rad} \mathrm{s}^{-1}, \omega_{r}=\omega_{0}, k^{2}=0.07, \xi=0.0156\right)$.

\subsection{Remarks about the results from the analytical model}

From the above investigations with the analytical model, one can infer that an optimal BSM generator for a selected excitation has to satisfy the following criteria: 
- Low damping ratio. Lower $\xi$ promises better displacement responses with wider operation bandwidth as shown in figure 7. Moreover, it multiplies the probabilities for the BSM generator to work at the high-energy orbits that is favorable to the available power.

- Properly high coupling level. Considering both the peak power and the average power over the interested frequency range, excessively high coupling level will introduce too much damping to the generator's dynamic responses. Thus, it decreases the available power as insufficient coupling cases unless the load varies correspondingly. It underlines that it is not necessary to realize a generator with a very high coupling level for the sake of the available power. It has to be pointed out that a high coupling level will improves the load independence properties.

- Matched electrical load. As the case for linear generators, there is an optimal load for the nonlinear BSM generator. However, the investigations show an interesting result that the average power merit used for this wideband generator does not strongly relies on the load. It means that the power performance is relatively stable if the load situation changes in a proper range.

- Optimal buckling level and low characteristic frequency. Buckling level $\varepsilon$ and characteristic frequency $\omega_{0}$ are the two most important structural parameters that determine the potential curve, including the potential barrier. The analysis on the proposed model confirms the existence of the optimal buckling level as shown in literature (Cottone et al., 2012; Cottone et al., 2009; Liu et al., 2015). Indicated by the studies, $\omega_{0}$ presents a different effect from $\varepsilon$ that the performance declines as $\omega_{0}$ value increases. Moreover, $\omega_{0}$ appears to have less influence on the power amplitude that is more determined by $\varepsilon$.

It is worthy of note that the optimal parameter might not be satisfied due to the structural limitations in most cases. Nevertheless, the discussions here can be a design guide to reach optimal BSM generators. However, this optimal performance is only accounted for the maximum available performance in the inter-well motion cases. In fact, if we calculate the average performance for both intra-well and inter-well cases, it shows that the inter-well case dominates the results while the intra-well case contributes only a small ratio.

\subsection{Short comments for the intra-well motion branch}

In real applications, the generator is likely to operate on the intra-well orbits that would lead to much smaller power output. Even though the low-level excitation makes the generator reside in a single potential well, the bandwidth widen effect is also observed as shown in figure 3. In this case, it is similar to a softening monostable generator.

The operation on high-energy inter-well orbits or low-energy intra-well orbits is dependent on the initial condition of the generator (Erturk et al., 2011), the attracting basin for different stable solutions (Daqaq et al., 2014) and the excitation (Liu et al., 2013a) etc. The common ways to make the generator switch from the low-energy orbit to the high-energy one are to increase the excitation force or decrease the potential barrier as discussed in section 4. Fortunately, more possible methods are envisioned in some literatures (Tang et al.,2010; Elvin and Erturk, 2013), such as using a virtual negative load (Masuda et al., 2013), applying a shock excitation (Wu et al., 2014), utilizing the stochastic resonance (Ramlan et al., 2010) or adaptively adjusting the bistable potential well (.Hosseinloo and Turitsyn, 2016). 


\section{Conclusion}

In this paper, we develop an analytical model dedicated to the BSM architecture generators using harmonic balance analysis. This simple modeling approach points out that the generator has some special properties especially that the piezoelectric transducer's deformation is not proportionally to the displacement as most generators. The electric charge accumulation rate on the piezoelectric transducer is no longer linearly dependent on the velocity, but determined by the product of the velocity and the displacement instead. In the intra-well motion case, this effect is negligible, but relevant in the inter-well motion case. Consequently, the usual method of considering energy harvesting as a constant viscous damping ratio is not suitable any more. According to the feature of the BSM generator, a doubled frequency voltage solution is assumed in the harmonic analysis. The newly derived analytical model has then been validated by matched experimental results. Besides, the conditions for intra-well motions to inter-well motions are discussed.

Compared with many proposed analytical models for the nonlinear generator, the proposed model in this article deals with several critical variables, which are related to the specific device parameters respectively ( $\omega_{0}$ the characteristic frequency, $k^{2}$ the electromechanical coupling coefficient, $\xi$ the mechanical damping ratio, $\varepsilon$ the buckling level and $\omega_{r}$ the electric load). These factors are well decoupled. Therefore, the optimizations of the generator for each parameter are feasible with this analytical model, especially considering the consistency between theory and experiment. All the critical parameters are investigated in detail using the available average power of the inter-well motions as the figure merit. It shows that an optimal generator is meant to have a low damping ratio, a properly high coupling level, a matched load, an optimal buckling level and a low characteristic frequency. In addition, some interesting results not emphasized before are presented here about the load, the coupling level and the characteristic frequency. It helps us to better understand the design of the BSM generator. Then we can conclude that the analytical model can provide an efficient and fast tool for the optimization and design of the BSM generator in real applications.

Further work will focus on developing an optimized design of the BSM generator with the help of the proposed analytical tool and exploring feasible structures and systems to facilitate the transition from the low-energy orbit to the high-energy one. This would allow more efficient and reliable devices to be obtained.

\section{Declaration of conflicting interests}

The authors declared no potential conflicts of interest with respect to the research, authorship, and/or publication of this article.

\section{Funding}

This work has been supported by the French National Research Agency (ANR) under grant No. ANR-11-JS09-002-01 (REVilaBa Project), the National Natural Science Foundation of China (No.51505395) and the Fundamental Research Funds for the Central Universities of China (A0920502051514-5). 


\section{References}

Arroyo E, Badel A and Formosa F (2013) Energy harvesting from ambient vibrations: Electromagnetic device and synchronous extraction circuit. J. Intell. Mater. Syst. Struct. 24(16):2023-2035.

Arroyo E, Badel A, Formosa F, Wu Y and Qiu J (2012) Comparison of electromagnetic and piezoelectric vibration energy harvesters: model and experiments. Sensors and Actuators A: Physical 183:148-156.

Challa VR, Prasad MG and Fisher FT (2011) Towards an autonomous self-tuning vibration energy harvesting device for wireless sensor network applications. Smart Materials and Structures 20(2):025004.

Cohen N, Bucher I and Feldman M (2012) Slow-fast response decomposition of a bi-stable energy harvester. Mechanical Systems and Signal Processing 31:29-39.

Cottone F, Gammaitoni L, Vocca H, Ferrari M and Ferrari V (2012) Piezoelectric buckled beams for random vibration energy harvesting. Smart Materials and Structures 21: 035021.

Cottone F, Vocca H and Gammaitoni L (2009) Nonlinear Energy Harvesting. Physical Review Letters 102(8):080601.

Chen LQ and Jiang WA (2015) Internal Resonance Energy Harvesting. Journal of Applied Mechanics, 82: 031004. http://doi.org/10.1115/1.4029606

Daqaq MF, Masana R., Erturk A and Dane Quinn D (2014) On the Role of Nonlinearities in Vibratory Energy Harvesting: A Critical Review and Discussion. Applied Mechanics Reviews, 66(4): 040801. http://doi.org/10.1115/1.4026278

Elvin N and Erturk A (2013) Advances in Energy Harvesting Methods. Springer.

Erturk A and Inman DJ (2011) Broadband piezoelectric power generation on high-energy orbits of the bistable Duffing oscillator with electromechanical coupling. Journal of Sound and Vibration 330(10):2339-2353.

Galchev T, Kim H and Najafi K (2011) Micro power generator for harvesting low-frequency and nonperiodic vibrations. Journal of Microelectromechanical Systems 20(4):852-866.

Hajati A and Kim S (2011) Ultra-wide bandwidth piezoelectric energy harvesting. Applied Physics Letters 99(8):083105.

Harb A (2011) Energy harvesting: State-of-the-art. Renewable Energy 36(10):2641-2654.

Harne RL and Wang KW (2013a) A review of the recent research on vibration energy harvesting via bistable systems. Smart Materials and Structures 22(2):023001.

Harne RL, Thota M and Wang KW (2013b) Bistable energy harvesting enhancement with an auxiliary linear oscillator. Smart Materials and Structures, 22(12): 125028. http://doi.org/10.1088/0964-1726/22/12/125028

Harne R and Wang K (2014) On the fundamental and superharmonic effects in bistable energy harvesting. Journal of Intelligent Material Systems and Structures 25(8):937-950.

Hosseinloo AH, and Turitsyn K (2016) Non-resonant energy harvesting via an adaptive bistable potential. Smart Materials and Structures, 25(1): 015010. http://doi.org/10.1088/0964-1726/25/1/015010

Ko JG, Lu C and Srivastava MB (2010) Wireless sensor networks for healthcare. Proceedings of the IEEE 98(11):1947-1960.

Kim P and Seok J (2014) A multi-stable energy harvester: Dynamic modeling and bifurcation analysis. Journal of Sound and Vibration, 333(21), 5525-5547. doi:10.1016/j.jsv.2014.05.054

Leland ES and Wright PK (2006) Resonance tuning of piezoelectric vibration energy scavenging generators using compressive axial preload. Smart Materials and Structures 15:1413-1420.

Liu H, Lee C, Kobayashi T, Tay CJ and Quan C (2012) Investigation of a MEMS piezoelectric energy harvester system with a frequency-widened-bandwidth mechanism introduced by mechanical stoppers. Smart Materials and Structures 21(3):035005. 
Liu WQ, Badel A, Formosa F, Wu Y and Agbossou A (2013a) Novel piezoelectric bistable oscillator architecture for wideband vibration energy harvesting. Smart Materials and Structures 22(3):035013.

Liu WQ, Badel A, Formosa F, Wu Y and Agbossou A (2013b) Wideband energy harvesting using a combination of an optimized synchronous electric charge extraction circuit and a bistable harvester. Smart Materials and Structures 22(12):125038.

Liu W, Formosa F, Badel A, Wu Y and Agbossou A (2014) Self-powered nonlinear harvesting circuit with a mechanical switch structure for a bistable generator with stoppers. Sensors and Actuators A: Physical 216: $106-115$.

Liu W, Badel A, Formosa F, Wu Y, Bencheikh N and Agbossou A (2015) A wideband integrated piezoelectric bistable generator: Experimental performance evaluation and potential for real environmental vibrations Journal of Intelligent Material Systems and Structures 26(7) 872-877.

Leadenham S and Erturk A (2015) Nonlinear M-shaped broadband piezoelectric energy harvester for very low base accelerations: primary and secondary resonances. Smart Materials and Structures, 24(5): 055021. http://doi.org/10.1088/0964-1726/24/5/055021

Mann BP and Sims ND (2009) Energy harvesting from the nonlinear oscillations of magnetic levitation. Journal of Sound and Vibration 319:515-530.

Mann BP and Owens BA (2010) Investigations of a nonlinear energy harvester with a bistable potential well. Journal of Sound and Vibration 329(9):1215-26.

Masana R and Daqaq MF (2011) Relative performance of a vibratory energy harvester in mono- and bistable potentials. Journal of Sound and Vibration 330(24):6036-6052.

Masuda A, Senda A, Sanada T and Sone A (2013) Global stabilization of high-energy response for a Duffing-type wideband nonlinear energy harvester via self-excitation and entrainment. Journal of Intelligent Material Systems and Structures 24(13):1598-1612.

Nayfeh AH and Mook DT (2008) Nonlinear oscillations. John Wiley \& Sons.

Neri I, Travasso F, Mincigrucci R, Vocca H, Orfei F and Gammaitoni L (2012) A real vibration database for kinetic energy harvesting application. Journal of Intelligent Material Systems and Structures 23(18):2095-2101.

Neiss S, Goldschmidtboeing F, Kroener M and Woias P (2014) Analytical model for nonlinear piezoelectric energy harvesting devices. Smart Materials and Structures 23(10):105031.

Nguyen SD and Halvorsen E (2011) Nonlinear springs for bandwidth-tolerant vibration energy harvesting. Journal of Microelectromechanical Systems 20(6):1225-1227.

Oliveira LM and Rodrigues JJ (2011) Wireless Sensor Networks: a Survey on Environmental Monitoring. Journal of Communications 6(2):143-151.

Owens BAM and Mann BP (2012) Linear and nonlinear electromagnetic coupling models in vibration-based energy harvesting. Journal of Sound and Vibration, 331(4): 922-937.

Pellegrini SP, Tolou N, Schenk M and Herder JL (2013) Bistable vibration energy harvesters: A review. Journal of Intelligent Material Systems and Structures 24(11):1303-1312.

Pillatsch P, Yeatman EM and Holmes AS (2012) A scalable piezoelectric impulse excited energy harvester for human body excitation. Smart Materials and Structures 21(11):115018.

Ramlan R, Brennan MJ, Mace BR and Kovacic I (2010) Potential benefits of a non-linear stiffness in an energy harvesting device. Nonlinear Dynamics 59(4): 545-558. doi:10.1007/s11071-009-9561-5.

Roundy S, Wright PK and Rabaey J (2003) A study of low level vibrations as a power source for wireless sensor nodes. Comput. Commun. 26:1131-1144. 
Roundy S and Zhang Y (2005) Toward self-tuning adaptive vibration based microgenerators. Proc. of SPIE 5649:373-384.

Ruiz-Garcia L, Lunadei L, Barreiro P and Robla JI (2009) A review of wireless sensor technologies and applications in agriculture and food industry: state of the art and current trends. Sensors (Basel, Switzerland) 9(6):4728-50.

Sebald G, Kuwano H, Guyomar D and Ducharne B (2011) Experimental Duffing oscillator for broadband piezoelectric energy harvesting. Smart Materials and Structures 20(10):102001.

Shahruz SM (2006) Design of mechanical band-pass filters for energy scavenging. Journal of Sound and Vibration 292:987-998.

Soliman MSM, Abdel-Rahman EM, El-Saadany EF and Mansour RR (2008) A wideband vibration-based energy harvester. Journal of Micromechanics and Microengineering 18(11):115021.

Stanton SC, McGehee CC and Mann BP (2009) Reversible hysteresis for broadband magnetopiezoelastic energy harvesting. Applied Physics Letters 95(17):174103.

Stanton SC, McGehee CC and Mann BP (2010) Nonlinear dynamics for broadband energy harvesting: Investigation of a bistable piezoelectric inertial generator. Physica D 239(10):640-53.

Stanton S, Owens B and Mann B (2012a) Harmonic balance analysis of the bistable piezoelectric inertial generator. Journal of Sound and Vibration 331(15):3317-3327.

Stanton SC, Mann BP and Owens BAM (2012b) Melnikov theoretic methods for characterizing the dynamics of the bistable piezoelectric inertial generator in complex spectral environments. Physica D 241(6):711-720.

Tang L, Yang Y and Soh CK (2010) Toward broadband vibration-based energy harvesting. Journal of Intelligent Material Systems and structures 21(18):1867.

Tang QC, Yang L and Li X (2011) Bi-stable frequency up-conversion piezoelectric energy harvester driven by non-contact magnetic repulsion. Smart Materials and Structures 20:125011.

Toprak A and Tigli O (2014) Piezoelectric energy harvesting: State-of-the-art and challenges. Applied Physics Reviews 1(3):031104. doi:10.1063/1.4896166.

Wickenheiser A M and Garcia E (2010) Broadband vibration-based energy harvesting improvement through frequency up-conversion by magnetic excitation Smart Materials and Structures 19(6):065020.

Wu Y, Badel A, Formosa F, Liu W and Agbossou A (2014) Nonlinear vibration energy harvesting device integrating mechanical stoppers used as synchronous mechanical switches. Journal of Intelligent Material Systems and Structures 25(14):1658-1663.

Xu JW, Liu YB, Shao WW and Feng Z (2012) Optimization of a right-angle piezoelectric cantilever using auxiliary beams with different stiffness levels for vibration energy harvesting. Smart Mater. Struct. 21(6):065017.

Yang Z and Yang J (2009) Connected vibrating piezoelectric bimorph beams as a wide-band piezoelectric power harvester. Journal of Intelligent Material Systems and Structures 20:569-574.

Zhu D, Tudor MJ and Beeby SP (2010) Strategies for increasing the operating frequency range of vibration energy harvesters: a review. Measurement Science and Technology 21(2):022001. 